\title{
Evidence in favor of single parton scattering mechanism in $\Upsilon$ and $D$ associated production at the LHC
}

\author{
A. V. Karpishkov, ${ }^{*}$ M. A. Nefedov, ${ }^{\dagger}$ and V. A. Saleev ${ }^{\ddagger}$ \\ Samara National Research University, Moskovskoe Shosse, 34, 443086 Samara, Russia
}

(Received 11 April 2019; published 23 May 2019)

\begin{abstract}
Associated production of prompt $\Upsilon(1 S)$ and $D^{+, 0}$ mesons has been proposed as a golden channel for the search of double parton scattering, because single parton scattering contribution to the cross section is believed to be negligible on the basis of leading-order calculations in the collinear parton model. We study this process in the leading-order approximation of the parton Reggeization approach. Hadronization of a $b \bar{b}$ pair into bottomonium states is described within the framework of the nonrelativistic QCD-factorization approach, while production of $D$ mesons is described in the fragmentation model with scale-dependent fragmentation functions. We have found good agreement with $\mathrm{LHCb}$ data for various normalized differential distributions, except for the case of spectra on azimuthal angle differences at the small $\Delta \varphi$ values. Crucially, the total cross section in our single parton scattering model accounts for more than one-half of observed cross sections, thus dramatically shrinking the room for the double parton scattering mechanism.
\end{abstract}

DOI: 10.1103/PhysRevD.99.096021

\section{INTRODUCTION}

Double parton scattering (DPS) mechanism attracts a lot of attention, both theoretically and experimentally. Recent advances in theory of DPS include computation [1] of nextto-leading-order (NLO) corrections to $1 \rightarrow 2$ splitting functions, contributing to the scale evolution of two parton collinear parton distribution functions (PDFs) and filling remaining gaps in the proof of the transverse momentum dependent-factorization theorem for the double Drell-Yan process [2].

While for single-scale observables, such as the total inclusive cross section of some hard process with hard scale $Q$, the DPS contribution is always suppressed by powers of $\Lambda^{2} / Q^{2}$, where $\Lambda$ is some typical hadronic scale, and the DPS contribution can be comparable with the single parton scattering contribution for the differential distributions in some regions of phase space [3]. In the DPS picture, longitudinal- and transverse-momentum correlations of small- $x$ partons, participating in two independent hard scatterings, are quickly washed out by effects of scale evolution of two parton PDFs [4], so that the double parton PDF effectively factorizes into a product of usual PDFs, leading to the "pocket-formula" description of DPS as

\footnotetext{
*karpishkoff@gmail.com

†nefedovma@gmail.com

saleev@samsu.ru
}

Published by the American Physical Society under the terms of the Creative Commons Attribution 4.0 International license. Further distribution of this work must maintain attribution to the author(s) and the published article's title, journal citation, and DOI. Funded by SCOAP .

$$
\sigma_{\mathrm{DPS}} \simeq \frac{\sigma_{\mathrm{SPS}}^{(1)} \times \sigma_{\mathrm{SPS}}^{(2)}}{\sigma_{\mathrm{eff}}},
$$

where $\sigma_{\mathrm{DPS}}$ is the DPS contribution to the production cross section of the pair of objects, $\sigma_{\mathrm{SPS}}^{(1,2)}$ is the single parton scattering (SPS) cross section of the production of one final-state object, and $\sigma_{\text {eff }}$ is the nonperturbative "effective cross section," which is typically assumed to be a universal energy-independent parameter. Equation (1) leads to flat distributions for observables correlating momenta of particles produced in independent hard scatterings, in particular, for the azimuthal angle $\Delta \varphi$ or rapidity difference $\Delta y$ between momenta of components of the pair. In contrast, the SPS contribution to differential distributions in the processes of pair production of jets, vector bosons, or mesons containing heavy flavors typically decreases rather steeply with increasing $|\Delta y|$ or $\Delta \varphi$, varying from $\Delta \varphi=\pi$ down to zero. Therefore, an overshoot of an experimentally observed cross section over the best available SPS predictions in the region $\Delta \varphi \rightarrow 0$ or $|\Delta y| \gg 1$ can be interpreted as a signature of DPS.

Another way to probe the DPS mechanism is to use the fact that, due to Eq. (1), the DPS cross section grows with energy roughly as a square of the SPS cross section, so at sufficiently high energy the power suppression by hard scale will be compensated by a quickly growing cross section and the DPS contribution will dominate. To observe this effect at available energies, one chooses the process for which the SPS cross section is as small as possible, due to suppression by high power of $\alpha_{s}$. The hard scale also should be small in comparison to the collision energy, so 
that one stays in the low- $x$ region, where Eq. (1) is applicable. Pair production of mesons containing $c$ and $b$ quarks seems to be an ideal playground for these kinds of studies. But one has to anticipate that, due to low hard scales and involvement of relatively poorly understood physics of hadronization, the SPS cross section could receive unexpectedly large higher-order QCD corrections, which could invalidate the assumption of negligibility of the SPS contribution. Moreover, at low $x$, plenty of phase space is available for emission of additional relatively hard partons, which will broaden the distributions in variables like $\Delta \varphi$ and $\Delta y$, thus mimicking the effects of DPS and making the extraction of the DPS signal from differential distributions more model dependent.

In Ref. [5], the strategy described in the previous paragraph has been applied by the LHCb collaboration for the search of DPS contribution in the process of associated production of prompt bottomonium $\Upsilon(1 S)$ and open-charm mesons $D^{0}$ or $D^{+}$in the forward-rapidity region in $p p$ collisions with center-of-mass energies $\sqrt{S}=7$ and $8 \mathrm{TeV}$ at the Large Hadron Collider. The leading-order [LO, $O\left(\alpha_{s}^{4}\right)$ in this case] calculations in the collinear parton model $[6,7]$ and color-singlet approximation for the hadronization of $b \bar{b} \rightarrow \Upsilon$ lead to a very small SPS total cross section of this process, compared with the cross section of inclusive production of $\Upsilon$ mesons, calculated in the same approximation: $R_{\mathrm{SPS}}^{(\mathrm{LO})}=\sigma_{\mathrm{SPS}}^{(\mathrm{LO}, \mathrm{CS})}(\Upsilon+c \bar{c}) / \sigma_{\mathrm{SPS}}^{(\mathrm{LO}, \mathrm{CS})}(\Upsilon)=(0.2-0.6) \%$, while experimentally this ratio reaches almost $8 \%$ at $\sqrt{S}=8 \mathrm{TeV}$ [5]. Such a large discrepancy was interpreted in Ref. [5] as a clear signal of a DPS mechanism. This interpretation was supported by the fact that shapes of differential distributions measured in Ref. [5] can be reasonably described by simple Monte Carlo calculations based on measured inclusive cross sections of production of $\Upsilon$ and $D$ mesons and the pocket formula (1).

In the present paper, we reconsider the estimation of SPS contribution to the above-described process, adding a few effects that were missed in calculations of Refs. [6,7]. First, we approximately take into account higher-order corrections coming from initial-state radiation (ISR) effects, by virtue of the parton Reggeization approach (PRA) [8,9], which allows us to factorize the most significant part of ISR corrections into unintegrated parton distribution functions consistently with QCD gauge invariance. The latter fact allows us to take into account the contributions to the $b \bar{b} \rightarrow \Upsilon$ coming from the color-octet $b \bar{b}$ pair in a framework of the nonrelativistic QCD (NRQCD) factorization approach [10]. An importance of color-octet contributions for bottomonium production in the PRA has been demonstrated by us in Ref. [11]. And finally, we consistently take into account parton to $D$ meson hadronization, using the scale-dependent fragmentation functions, obtained in a global analysis of open-charm hadron production in $e^{+} e^{-}$-annihilation in Ref. [12]. Counterintuitively, not only $c \rightarrow D$ but also $g \rightarrow D$ fragmentation plays an important role in the associated $\Upsilon$ and $D$ production. Gluon fragmentation has also been found to be important for the samesign $D D$ pair production in Refs. [13,14]. In total and taking into account the uncertainties, our hybrid PRA+ NRQCD+fragmentation model can account for more than half of the experimentally observed cross section for $\Upsilon D$ pair production. The shapes of all differential distributions in our SPS model also turn out to reproduce experimental data rather well, except for the shape of the $\Delta \varphi$ spectrum which has a puzzling "upside-down" behavior, unexplainable even by the DPS model (1). Therefore, we show that radiative corrections to the SPS cross section of the process under consideration are large and mimic the behavior of DPS, thus weakening the case for DPS dominance in this process.

The paper is organized as follows. We describe the basics of the PRA in Sec. II. In Sec. III, we present our model of $\Upsilon(1 S) D^{0,+}$ pair production. Then we concentrate on the numerical results and comparison with the experimental data of Ref. [5] in Sec. IV. Finally, we summarize our conclusions in Sec. V.

\section{PARTON REGGEIZATION APPROACH}

A brief description of LO approximation of the PRA is presented below. More details can be found in Ref. [9], the development of the PRA in the NLO approximation is further discussed in $[15,16]$. The main ingredients of the PRA are the $k_{T}$-dependent factorization formula, unintegrated parton distribution functions, and gauge-invariant amplitudes with off-shell initial-state partons, derived using Lipatov's effective field theory (EFT) of Reggeized gluons [17] and Reggeized quarks [18].

The factorization formula of the PRA in LO approximation for the process $p+p \rightarrow \mathcal{Y}+X$ can be obtained from the factorization formula of the collinear parton model for the auxiliary hard subprocess $g+g \rightarrow g+\mathcal{Y}+g$. In Ref. [9], the modified multi-Regge-kinematics (MMRK) approximation for the auxiliary amplitude is constructed, which correctly reproduces the multi-Regge and collinear limits of the corresponding QCD amplitude. This MMRK amplitude has a $t$-channel factorized form, which allows one to rewrite the cross section of the auxiliary subprocess in a $k_{T}$-factorized form

$$
\begin{aligned}
d \sigma= & \int_{0}^{1} \frac{d x_{1}}{x_{1}} \int \frac{d^{2} \mathbf{q}_{T 1}}{\pi} \tilde{\Phi}_{g}\left(x_{1}, t_{1}, \mu^{2}\right) \\
& \times \int_{0}^{1} \frac{d x_{2}}{x_{2}} \int \frac{d^{2} \mathbf{q}_{T 2}}{\pi} \tilde{\Phi}_{g}\left(x_{2}, t_{2}, \mu^{2}\right) \cdot d \hat{\sigma}_{\mathrm{PRA}},
\end{aligned}
$$

where $t_{1,2}=-\mathbf{q}_{T 1,2}^{2}$, and the off-shell partonic cross section $\hat{\sigma}_{\text {PRA }}$ in the PRA is determined by squared PRA amplitude, $\overline{\left|\mathcal{A}_{\mathrm{PRA}}\right|^{2}}$. Despite the fact that four-momenta $\left(q_{1,2}\right)$ of partons in the initial state of amplitude $\mathcal{A}_{\mathrm{PRA}}$ are off shell 
$\left(q_{1,2}^{2}=-t_{1,2}<0\right)$, the PRA hard-scattering amplitude is gauge invariant because the initial-state off-shell gluons are treated as Reggeized gluons $(R)$ in a sense of gaugeinvariant EFT for QCD processes in multi-Regge kinematics (MRK), introduced by Lipatov in [17]. The Feynman rules of this EFT are written down in Ref. [19].

The tree-level "unintegrated PDFs" $\tilde{\Phi}_{g}\left(x_{1,2}, t_{1,2}, \mu^{2}\right)$ in Eq. (2) are equal to the convolution of the collinear PDF $f_{g}\left(x, \mu^{2}\right)$ and Dokshitzer-Gribov-Lipatov-AltarelliParisi splitting function $P_{g g}(z)$ with the factor $1 / t_{1,2}$. Consequently, the cross section (2) with such unintegrated PDFs contains the collinear divergence at $t_{1,2} \rightarrow 0$ and infrared divergence at $z_{1,2} \rightarrow 1$. To regularize the latter, we observe that the MMRK expression gives a reasonable approximation for the exact matrix element only in the rapidity-ordered part of the phase space $y_{g_{1}}>y_{\mathcal{Y}}>y_{g_{2}}$. From this requirement, the following cutoff on $z_{1,2}$ can be derived: $z_{1,2}<1-\Delta_{\mathrm{KMR}}\left(t_{1,2}, \mu^{2}\right)$, where $\Delta_{\mathrm{KMR}}\left(t, \mu^{2}\right)=$ $\sqrt{t} /\left(\sqrt{\mu^{2}}+\sqrt{t}\right)$ is the Kimber-Martin-Ryskin (KMR) cutoff function [20], and we have taken into account that $\mu^{2} \sim M_{T \mathcal{Y}}^{2}$. The collinear singularity at $t_{1,2} \rightarrow 0$ is regularized by the Sudakov form factor

$$
\begin{aligned}
T_{i}\left(t, \mu^{2}\right)= & \exp \left[-\int_{t}^{\mu^{2}} \frac{d t^{\prime}}{t^{\prime}} \frac{\alpha_{s}\left(t^{\prime}\right)}{2 \pi}\right. \\
& \left.\times \sum_{j=q, \bar{q}, g} \int_{0}^{1} d z z \cdot P_{j i}(z) \theta\left(1-\Delta_{\mathrm{KMR}}\left(t^{\prime}, \mu^{2}\right)-z\right)\right],
\end{aligned}
$$

which resums doubly logarithmic corrections $\sim \log ^{2}\left(t / \mu^{2}\right)$ in the leading-logarithmic approximation.

The final form of our unintegrated PDF in the PRA is

$$
\begin{aligned}
\Phi_{i}\left(x, t, \mu^{2}\right)= & \frac{T_{i}\left(t, \mu^{2}\right)}{t} \frac{\alpha_{s}(t)}{2 \pi} \\
& \times \sum_{j=q, \bar{q}, g} \int_{x}^{1} d z P_{i j}(z) \cdot \frac{x}{z} f_{j}\left(\frac{x}{z}, t\right) \\
& \cdot \theta\left(1-\Delta_{\mathrm{KMR}}\left(t, \mu^{2}\right)-z\right),
\end{aligned}
$$

which coincides with Kimber, Martin, and Ryskin unintegrated PDF [20]. The KMR unintegrated PDF is actively used in phenomenological studies employing $k_{T}$ factorization, but to our knowledge, the derivation presented in [9] is the first systematic attempt to clarify its relationships with the MRK limit of the QCD amplitudes.

In contrast to most of studies in $k_{T}$ factorization, the gauge-invariant matrix elements with off-shell initial-state partons (Reggeized quarks and gluons) from Lipatov's EFT $[17,18]$ allow one to study arbitrary processes involving the non-Abelian structure of QCD without violation of Slavnov-Taylor identities due to the nonzero virtuality of initial-state partons. This approach, together with KMR unintegrated PDFs gives stable and consistent results in a wide range of phenomenological applications, which include the description of the angular correlations of dijets [8], $b$ jets [21], charmed [14], and bottom-flavored [9] mesons, as well as some other examples.

A few years ago, the new approach to derive gaugeinvariant scattering amplitudes with off-shell initial-state partons for high-energy scattering, using the spinor-helicity techniques and Britto-Cachazo-Feng-Witten-like recursion relations for such amplitudes, was introduced in Refs. [2224]. This formalism for numerical generation of off-shell amplitudes is equivalent to Lipatov's EFT at the tree level, but for some observables, e.g., related with production of heavy quarkonia, or for the generalization of the formalism to NLO $[15,16]$, the use of explicit Feynman rules and the structure of EFT is more convenient.

\section{MODEL FOR YD PAIR PRODUCTION}

In the leading order $O\left(\alpha_{s}^{3}\right)$ in the PRA plus fragmentation model [25], only gluon fragmentation contributes to the process of associated production of bottomonium and $D$ mesons

$$
\begin{gathered}
R+R \rightarrow \Upsilon(3 S, 2 S, 1 S)+g(\rightarrow D), \\
R+R \rightarrow \chi_{b}(2 P, 1 P)+g(\rightarrow D) .
\end{gathered}
$$

One might worry that the gain of one power of $\alpha_{s}$ will be compensated by a small numerical value of the $g \rightarrow D$ fragmentation function in comparison with $c \rightarrow D$ fragmentation and thus the numerically leading contribution comes from the NLO $O\left(\alpha_{s}^{4}\right)$ subprocesses

$$
\begin{gathered}
R+R \rightarrow \Upsilon(3 S, 2 S, 1 S)+c(\rightarrow D)+\bar{c}, \\
R+R \rightarrow \chi_{b}(2 P, 1 P)+c(\rightarrow D)+\bar{c} .
\end{gathered}
$$

We address this question in Sec. IV and show that contribution of $2 \rightarrow 3$ subprocesses is actually subleading, so in the final predictions for the cross sections and spectra we take into account only subprocesses (5) and (6).

According to NRQCD factorization formalism [10], final heavy quarkonium can be produced via color-singlet and color-octet intermediate states of a $b \bar{b}$ pair. We use the set of color-singlet and color-octet nonperturbative (long-distance) matrix elements (NMEs), which has been obtained in the LO PRA plus NRQCD-factorization approximation in Ref. [11] from the fit of inclusive $p_{T}$ spectra of prompt $\Upsilon(n S)$ mesons, measured by ATLAS, CMS, and LHCb collaborations at the LHC. For the reader's convenience, we collect these NMEs in Table I. The color-octet contributions for the production of $P$-wave bottomonium tend to be negligible in comparison with color-singlet contributions [11], so in the $P$-wave case we take into account only the singlet channel. Thus, at the quark level, we are left with the following list of LO partonic subprocesses: 
TABLE I. The color-singlet and color-octet NMEs from Ref. [11] used in the calculation.

\begin{tabular}{|c|c|}
\hline NME & Fit in LO PRA \\
\hline$\left\langle\mathcal{O}^{\Upsilon(1 S)}\left[{ }^{3} S_{1}^{(1)}\right]\right\rangle \times \mathrm{GeV}^{-3}$ & 9.28 \\
\hline$\left\langle\mathcal{O}^{\mathrm{r}(1 S)}\left[{ }^{3} S_{1}^{(8)}\right]\right\rangle \times 10^{2} \mathrm{GeV}^{-3}$ & $2.31 \pm 0.25$ \\
\hline$\left\langle\mathcal{O}^{\Upsilon(1 S)}\left[{ }^{1} S_{0}^{(8)}\right]\right\rangle \times 10^{2} \mathrm{GeV}^{-3}$ & $0.0 \pm 0.05$ \\
\hline$\left\langle\mathcal{O}^{\Upsilon(1 S)}\left[{ }^{3} P_{0}^{(8)}\right]\right\rangle \times 10^{2} \mathrm{GeV}^{-5}$ & $0.0 \pm 0.38$ \\
\hline$\left\langle\mathcal{O}^{\mathrm{r}(2 S)}\left[{ }^{3} S_{1}^{(1)}\right]\right\rangle \times \mathrm{GeV}^{-3}$ & 4.62 \\
\hline$\left\langle\mathcal{O}^{\Upsilon}(2 S)\left[{ }^{3} S_{1}^{(8)}\right]\right\rangle \times 10^{2} \mathrm{GeV}^{-3}$ & $1.51 \pm 0.17$ \\
\hline$\left\langle\mathcal{O}^{\Upsilon(2 S)}\left[{ }^{1} S_{0}^{(8)}\right]\right\rangle \times 10^{2} \mathrm{GeV}^{-3}$ & $0.0 \pm 0.01$ \\
\hline$\left\langle\mathcal{O}^{\Upsilon}(2 S)\left[{ }^{3} P_{0}^{(8)}\right]\right\rangle \times 10^{2} \mathrm{GeV}^{-5}$ & $0.0 \pm 0.03$ \\
\hline$\left\langle\mathcal{O}^{\mathrm{r}(3 S)}\left[{ }^{3} S_{1}^{(1)}\right]\right\rangle \times \mathrm{GeV}^{-3}$ & 3.54 \\
\hline$\left\langle\mathcal{O}^{\Upsilon(3 S)}\left[{ }^{3} S_{1}^{(8)}\right]\right\rangle \times 10^{2} \mathrm{GeV}^{-3}$ & $1.24 \pm 0.13$ \\
\hline$\left\langle\mathcal{O}^{\Upsilon(3 S)}\left[{ }^{1} S_{0}^{(8)}\right]\right\rangle \times 10^{2} \mathrm{GeV}^{-3}$ & $0.0 \pm 0.01$ \\
\hline$\left\langle\mathcal{O}^{\Upsilon(3 S)}\left[{ }^{3} P_{0}^{(8)}\right]\right\rangle \times 10^{2} \mathrm{GeV}^{-5}$ & $0.0 \pm 0.02$ \\
\hline$\left\langle\mathcal{O}^{\chi(1 P)}\left[{ }^{3} P_{0}^{(1)}\right]\right\rangle \times \mathrm{GeV}^{-5}$ & 2.03 \\
\hline$\left\langle\mathcal{O}^{\chi(1 P)}\left[{ }^{3} S_{1}^{(8)}\right]\right\rangle \times 10^{2} \mathrm{GeV}^{-3}$ & 0.0 \\
\hline$\left\langle\mathcal{O}^{\chi(2 P)}\left[{ }^{3} P_{0}^{(1)}\right]\right\rangle \times \mathrm{GeV}^{-5}$ & 2.36 \\
\hline$\left\langle\mathcal{O} \chi(2 P)\left[{ }^{3} S_{1}^{(8)}\right]\right\rangle \times 10^{2} \mathrm{GeV}^{-3}$ & 0.0 \\
\hline
\end{tabular}
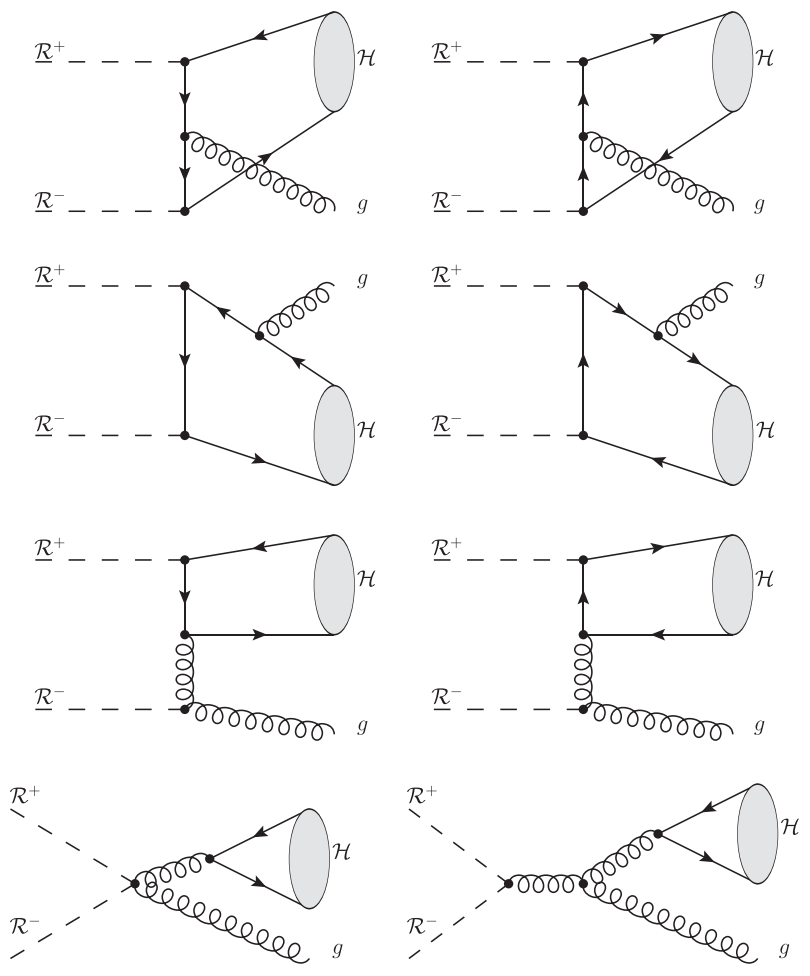

$$
\begin{gathered}
R+R \rightarrow b \bar{b}\left[{ }^{3} S_{1}^{(1)}\right]+g, \\
R+R \rightarrow b \bar{b}\left[{ }^{3} S_{1}^{(8)}\right]+g, \\
R+R \rightarrow b \bar{b}\left[{ }^{3} P_{0,1,2}^{(1)}\right]+g .
\end{gathered}
$$

The sets of Feynman diagrams of Lipatov's EFT that describe subprocesses (9)-(11) are shown in Fig. 1. The squared off-shell amplitude of subprocess (9) is well known; it was calculated many years ago in Ref. [27]. Squared offshell amplitudes of subprocess (10) and (11) are calculated here for the first time. However, corresponding on-shell squared amplitudes are known [28] and they have been used for the test of the collinear limit of obtained PRA amplitudes with off-shell initial-state partons. To automatize the analytic calculations, we have implemented the Feynman rules of EFT [17,18] as a model for the FEYNARTS package [29]. Further calculation of helicity amplitudes for $2 \rightarrow 2$ and $2 \rightarrow 3$ processes has been performed using the FeYNCALC [30] package and for the numerical evaluation, amplitudes have been squared, summed over colors and helicities, and implemented as a FORTRAN code. Unfortunately, due to complicated dependence on light cone and transverse components of four-momenta of initial and final-state particles, PRA amplitudes even for
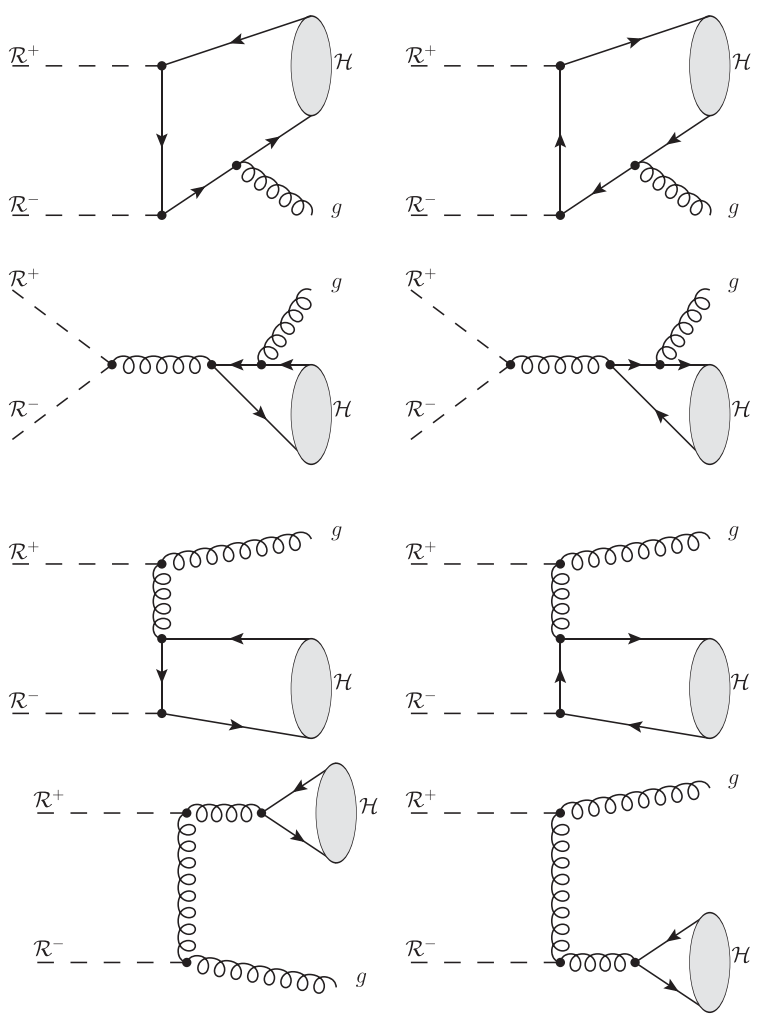

FIG. 1. Feynman diagrams used for direct production of $S$ and $P$ wave bottomonium via color-singlet and color-octet intermediate states in processes $R+R \rightarrow b \bar{b}\left[{ }^{3} S_{1}^{(1,8)}\right]+g$ and $R+R \rightarrow b \bar{b}\left[{ }^{3} P_{0,1,2}^{(1)}\right]+g$. 

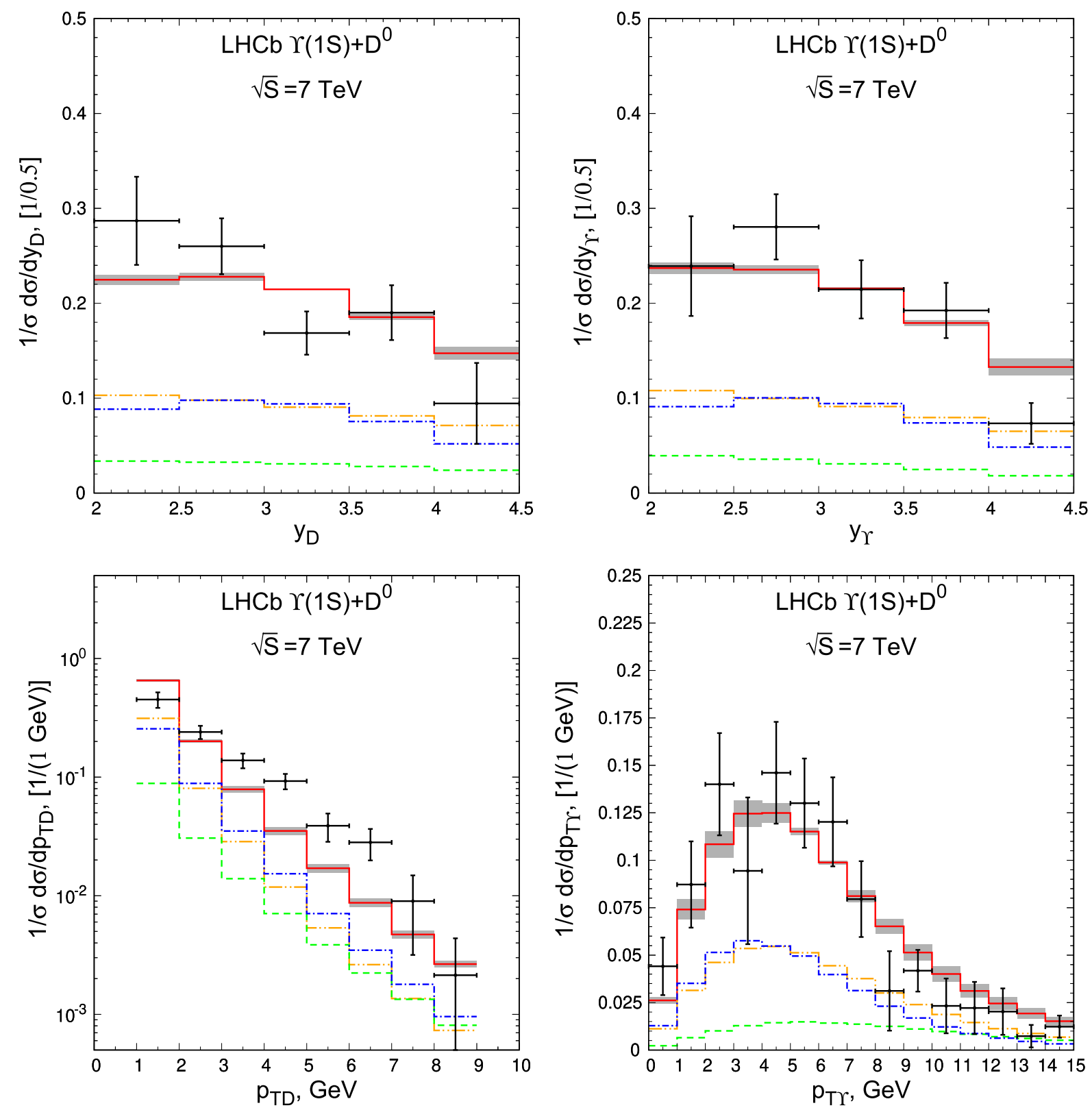

FIG. 2. Transverse momentum and rapidity spectra of $\Upsilon(1 S)$ and $D^{0}$. The LHCb data [5] are taken at $\sqrt{S}=7 \mathrm{TeV}$, $2.0<y_{\Upsilon(D)}<4.5,0<p_{T \Upsilon}<15 \mathrm{GeV}$, and $1<p_{T D}<20 \mathrm{GeV}$. Blue histograms are color-singlet contributions, green are coloroctet contributions, orange are the sum of feed-down contributions, red are the sum of all contributions.

$2 \rightarrow 2$ processes tend to become prohibitively large for the journal publication. In fact, in the case of ${ }^{3} S_{1}^{(8)}$ subprocess (10), the arithmetic with quadruple precision inside the squared-amplitude routine is required to reach numerical stability of the calculation.

Considering the associated production of $\Upsilon(1 S) D^{0,+}$ pairs, one should take into account both direct and feeddown production of $\Upsilon(1 S)$ via decays of higher-lying $\Upsilon(3 S, 2 S)$ and $\chi_{b}(2 P, 1 P)$ states, which in turn are produced directly or via decays of even excited states. Taking known branching fractions of different decays from [31] and considering cascades of up to three consequent decays, we have obtained the following cascade branching fractions for the $\Upsilon(1 S)$ state: $\operatorname{Br}(\Upsilon(3 S) \rightarrow \Upsilon(1 S))=0.138$, $\operatorname{Br}\left(\chi_{b 2}(2 P) \rightarrow \Upsilon(1 S)\right)=0.114, \operatorname{Br}\left(\chi_{b 1}(2 P) \rightarrow \Upsilon(1 S)\right)=$ $0.171, \quad \operatorname{Br}\left(\chi_{b 0}(2 P) \rightarrow \Upsilon(1 S)\right)=0.023, \quad \operatorname{Br}(\Upsilon(2 S) \rightarrow$ $\Upsilon(1 S))=0.302, \quad \operatorname{Br}\left(\chi_{b 2}(1 P) \rightarrow \Upsilon(1 S)\right)=0.191$, $\operatorname{Br}\left(\chi_{b 1}(1 P) \rightarrow \Upsilon(1 S)\right)=0.339, \quad$ and $\quad \operatorname{Br}\left(\chi_{b 0}(2 P) \rightarrow\right.$ $\Upsilon(1 S))=0.018$, which are used in the calculation.

To describe the $D$ meson production we use the fragmentation model in which the transition of gluon to the $D$ meson is described by the corresponding scaledependent fragmentation function (FF) $D_{g}\left(z, \mu^{2}\right)$ [25]. Recently, the nontrivial role of gluon fragmentation in 

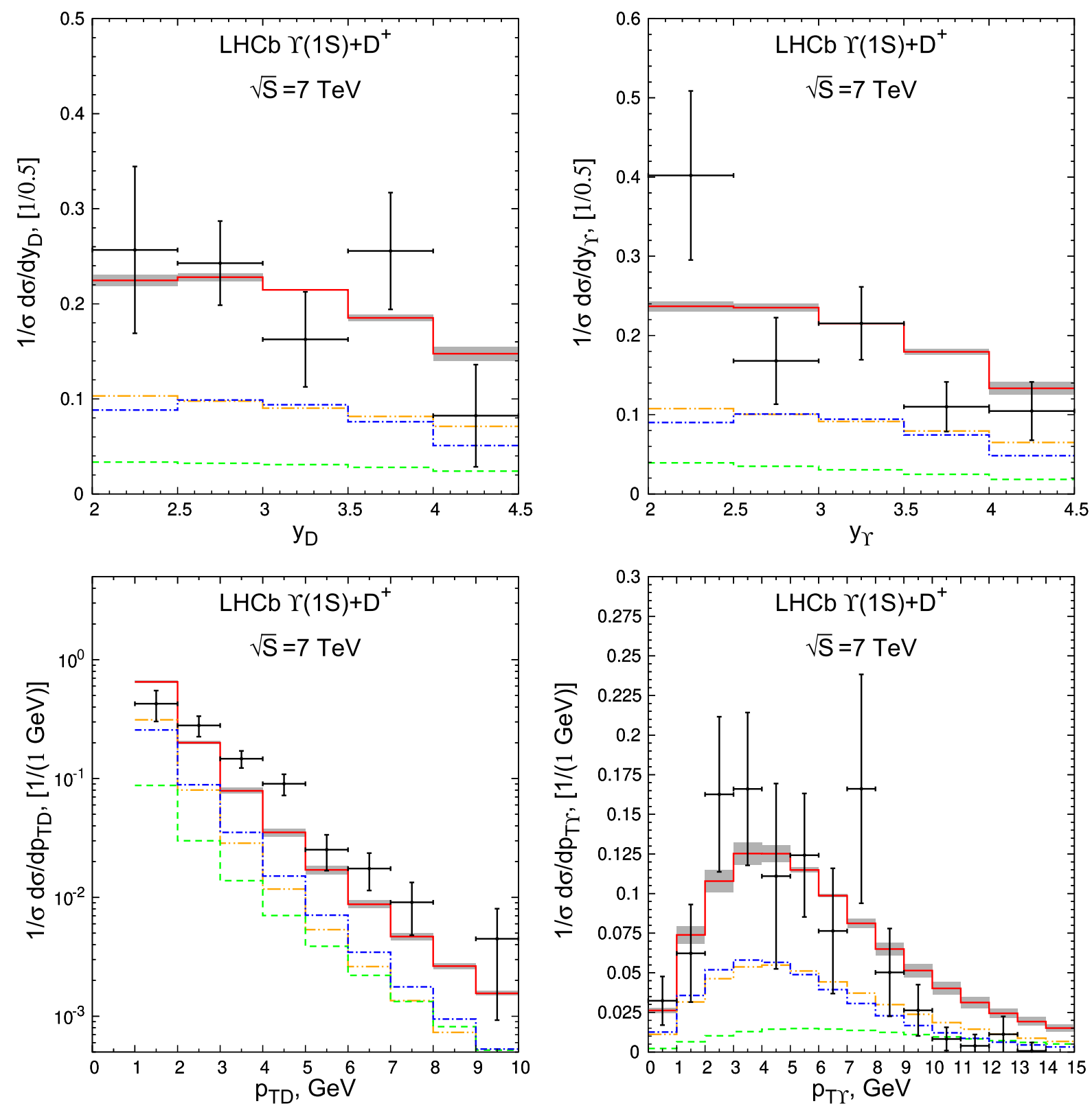

FIG. 3. Transverse momentum and rapidity spectra of $\Upsilon(1 S)$ and $D^{+}$. LHCb data [5] are taken at $\sqrt{S}=7 \mathrm{TeV}, 2.0<y_{\Upsilon(D)}<4.5$, $0<p_{T r}<15 \mathrm{GeV}$, and $1<p_{T D}<20 \mathrm{GeV}$. Blue histograms are color-singlet contributions, green are color-octet contributions, orange are the sum of feed-down contributions, red are the sum of all contributions.

associated production of same-sign $D$ meson pairs at the LHC has been demonstrated by some of us [14]. The latter process has been considered by some authors as a clean signal of the DPS production mechanism [26]. In Ref. [14], it has been shown that the main mechanism of same-sign $D D$ pair production is gluon to $D$ meson fragmentation via production of a gluon pair in the LO PRA subprocess $R R \rightarrow g g$. The description of LHCb data [32] has been archived without hypothesis on large DPS contribution. The present paper, as well as Ref. [14], uses universal scaledependent LO FFs of Ref. [12], fitted to $e^{+} e^{-}$-annihilation data from CERN LEP1.

\section{RESULTS AND DISCUSSION}

Here we discuss our numerical results obtained for prompt $\Upsilon(1 S) D^{0,+}$ pair production in $p p$ collisions at energies $\sqrt{S}=7$ and $\sqrt{S}=8 \mathrm{TeV}$. We use the unintegrated PDFs obtained by the KMR formula (3) and (4) from the LO collinear PDFs MSTW-2008 [33] and the corresponding value of $\alpha_{s}\left(M_{Z}\right)=0.13939$. We set the renormalization and factorization scales to $\mu_{R}=\mu_{F}=\frac{\xi}{2}\left(\sqrt{M_{\Upsilon}^{2}+p_{T \Upsilon}^{2}}+\right.$ $\left.\sqrt{M_{D}^{2}+p_{T D}^{2}}\right)$, where $\xi=1$ for the central lines of our predictions, and we vary $1 / 2<\xi<2$ to estimate the scale uncertainty of our prediction, which is shown in the figures 

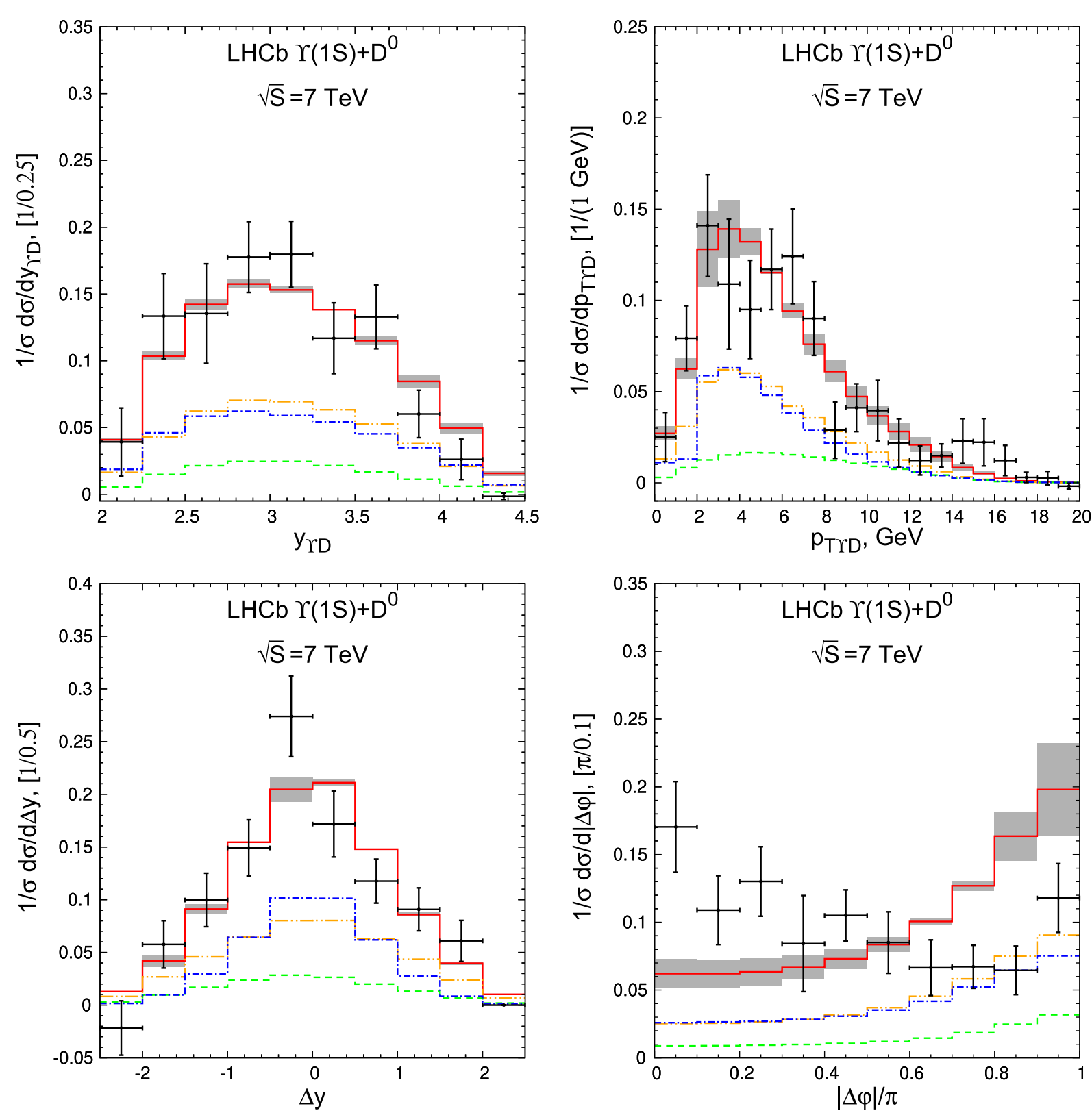

FIG. 4. (Top) Transverse momentum and rapidity spectra of $\Upsilon(1 S) D^{0}$ pairs. (Bottom) Rapidity difference and azimuthal angle difference spectra. Histograms are the same as in Fig. 2. LHCb data [5] are taken at $\sqrt{S}=7 \mathrm{TeV}, 2.0<y_{\Upsilon(D)}<4.5$, $0<p_{T \Upsilon}<15 \mathrm{GeV}$, and $1<p_{T D}<20 \mathrm{GeV}$.

by the gray band for the curve corresponding to the sum of all contributions.

In Figs. 2 and 3, we compare normalized transverse momentum and rapidity spectra of $\Upsilon(1 S)$ and $D^{0,+}$ predicted by our model with LHCb data [5]. The top panels of Figs. 4 and 5 collect rapidity and transversemomentum spectra of $\Upsilon(1 S) D^{0,+}$ pairs. For all abovementioned spectra, our hybrid LO PRA plus NRQCD plus fragmentation model does reasonably well in explaining the experimental data.

In the bottom panels of Figs. 4 and 5 we plot spectra for the rapidity difference $\Delta y=\left|y_{\Upsilon}-y_{D}\right|$ and for the azimuthal angle difference $\Delta \varphi=\left|\varphi_{\Upsilon}-\varphi_{D}\right|$. The $\Delta y$ spectra are reasonably well descried. The $\Delta \varphi$ spectrum obtained in our model has a typical shape for this kind of spectra. It has one peak at the $\Delta \varphi \rightarrow \pi$ and plateau at the $\Delta \varphi \leq \pi / 2$. However, the experimental data from LHCb collaboration, though having large errors, demonstrate existence of the second peak for $\Delta \varphi \rightarrow 0$. This feature of the data cannot be explained even by the DPS model based on Eq. (1), which predicts the flat, uncorrelated $\Delta \varphi$ spectrum.

In Figs. 6 and 7, we plot spectra for the invariant mass of $\Upsilon(1 S) D^{0,+}$ pair $(M)$ and transverse-momentum asymmetry 

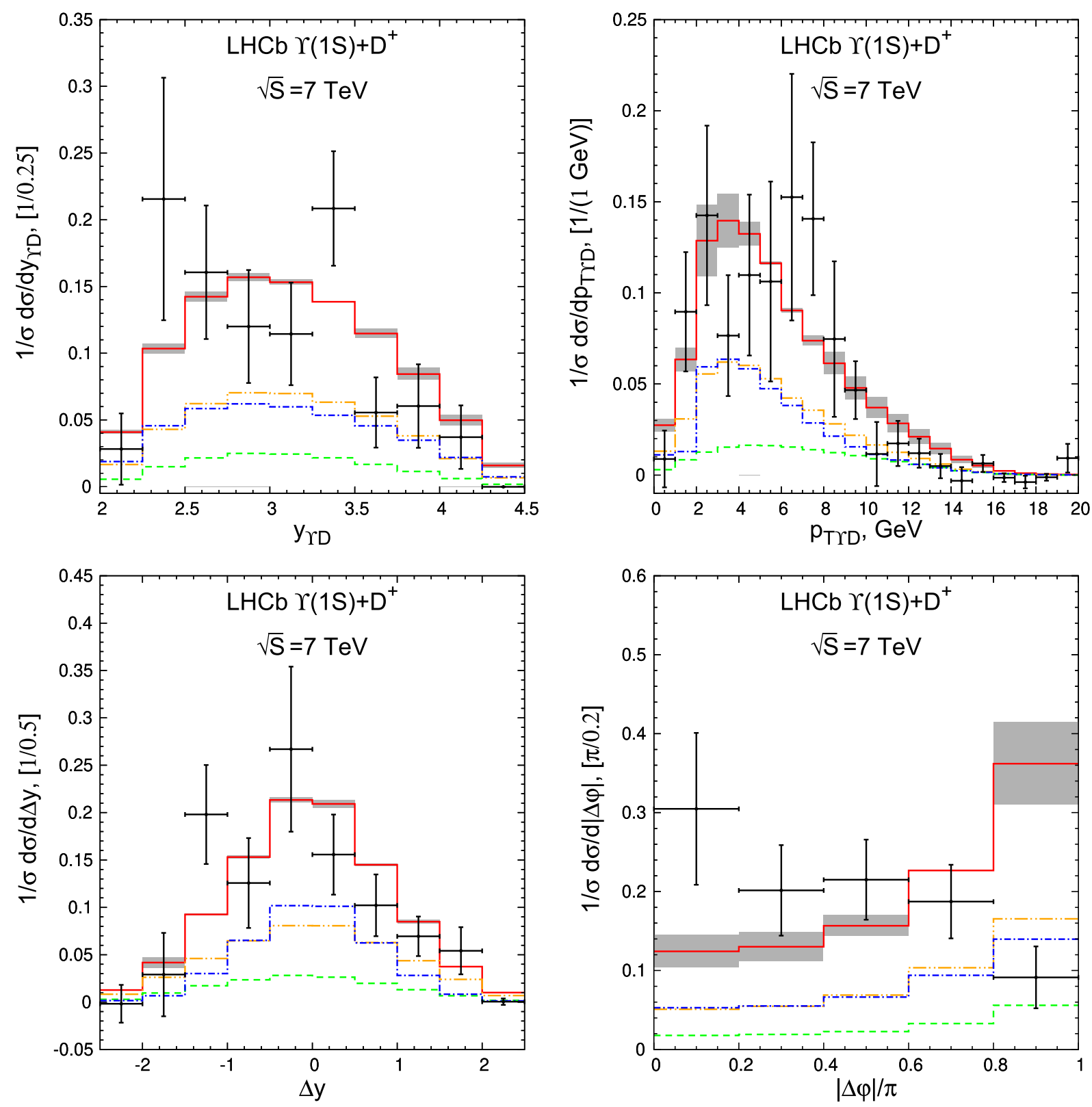

FIG. 5. (Top) Transverse momentum and rapidity spectra of $\Upsilon(1 S) D^{+}$pairs. (Bottom) Rapidity difference and azimuthal angle difference spectra. Histograms are the same as in Fig. 2. LHCb data [5] are taken at $\sqrt{S}=7 \mathrm{TeV}, 2.0<y_{\Upsilon(D)}<4.5$, $0<p_{T \Upsilon}<15 \mathrm{GeV}$, and $1<p_{T D}<20 \mathrm{GeV}$.

$A_{T}=\left(p_{T \Upsilon}-p_{T D}\right) /\left(p_{T \Upsilon}+p_{T D}\right)$. Predictions of our model with this correlation spectra also agree reasonably well with $\mathrm{LHCb}$ data.

Now we turn to the discussion of predictions of our SPS model for total cross sections $B_{\mu^{+} \mu^{-}} \times \sigma^{\Upsilon D^{0,+}}$ [where $\Upsilon(1 S) \rightarrow \mu^{+} \mu^{-}$branching fraction $B_{\mu^{+} \mu^{-}}=0.025$ is taken into account]. At $\sqrt{S}=7 \mathrm{TeV}$, central values of our predictions for $\Upsilon(1 S) D^{0}$ and $\Upsilon(1 S) D^{+}$production are 91 and $36 \mathrm{pb}$, respectively (Table II), which, especially taking into account large scale uncertainty, reaches almost more than one-half of the experimental cross section, which is, respectively, 155 and $82 \mathrm{pb}$. Different contributions to the total cross section and its scale uncertainty are presented in Tables II and III, correspondingly, for the energies $\sqrt{S}=7$ and $\sqrt{S}=8 \mathrm{TeV}$. The feeddown contribution from decays of higher lying bottomonium states $\left[\Upsilon(3 S), \Upsilon(2 S), \chi_{b}(2 P), \chi_{b}(1 P)\right]$ is not small; it is about $50 \%$ from the total calculated cross section. The contribution of color-singlet processes (5) and (6) is always dominant, but the contribution of the $\left[{ }^{3} S_{1}^{(8)}\right]$ intermediate state is also very important, reaching up to a half of the $\left[{ }^{3} S_{1}^{(1)}\right]$ intermediate-state contribution. 

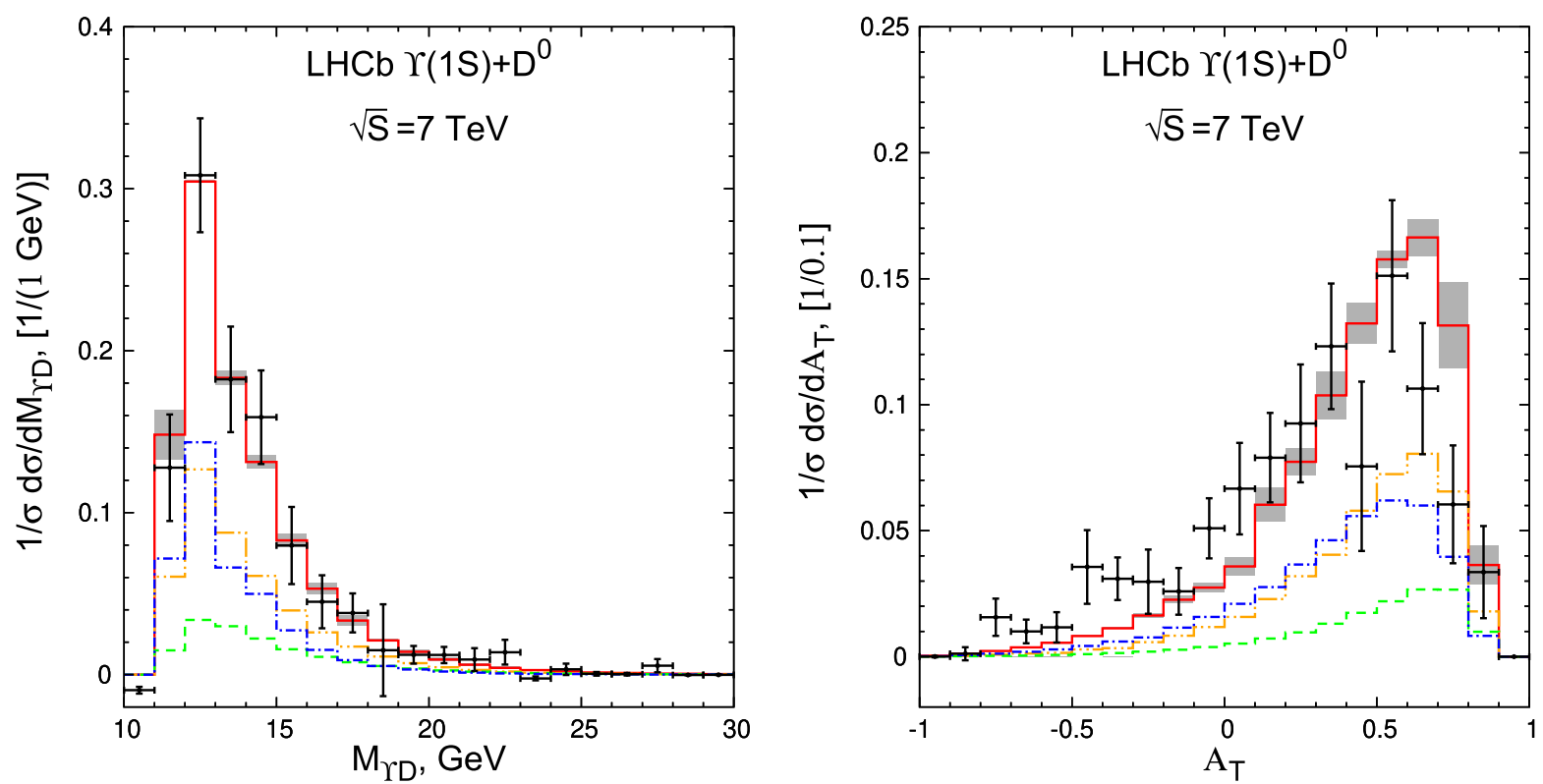

FIG. 6. (Left) Invariant mass spectrum of $\Upsilon(1 S) D^{0}$ pairs. (Right) Transverse-momentum asymmetry $\mathcal{A}_{T}$ spectrum. Histograms are the same as in Fig. 2. LHCb data [5] are taken at $\sqrt{S}=7 \mathrm{TeV}, 2.0<y_{\Upsilon(D)}<4.5,0<p_{T \Upsilon}<15 \mathrm{GeV}$, and $1<p_{T D}<20 \mathrm{GeV}$.

In our model, the main source of $D^{0,+}$ mesons is the gluon fragmentation. Let us compare the above results with the model based on $c$ quark fragmentation into $D$ mesons. We have calculated direct production cross sections via color-singlet and color-octet intermediate states in process (7), which on the quark level corresponds to

$$
R+R \rightarrow b \bar{b}\left[{ }^{3} S_{1}^{(1,8)}\right]+c+\bar{c}
$$

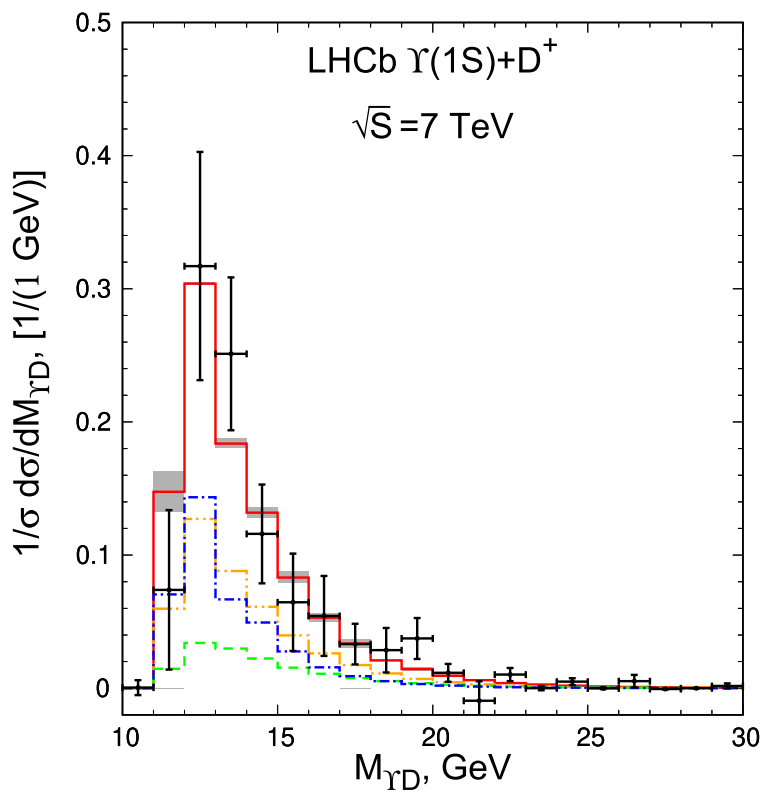

Due to the fragmentation model, we have to take the $c$ quark as massless, however, we take into account the threshold condition for the invariant mass of the $c \bar{c}$ pair: $s_{c \bar{c}}=\left(p_{c}+p_{\bar{c}}\right)^{2}>4 m_{c}^{2}$ with $m_{c}=1.5 \mathrm{GeV}$. Our $2 \rightarrow 3$ amplitudes passed numerous cross-checks; in particular, we have numerically checked that the final-state collinear limit $\left[s_{c \bar{c}} \ll \min \left(p_{T c}^{2}, p_{T \bar{c}}^{2}\right)\right]$ of the squared amplitudes of the processes (12) is related to the squared amplitudes of the processes (9) and (10) by the following well-known collinear-factorization relation for tree-level amplitudes:

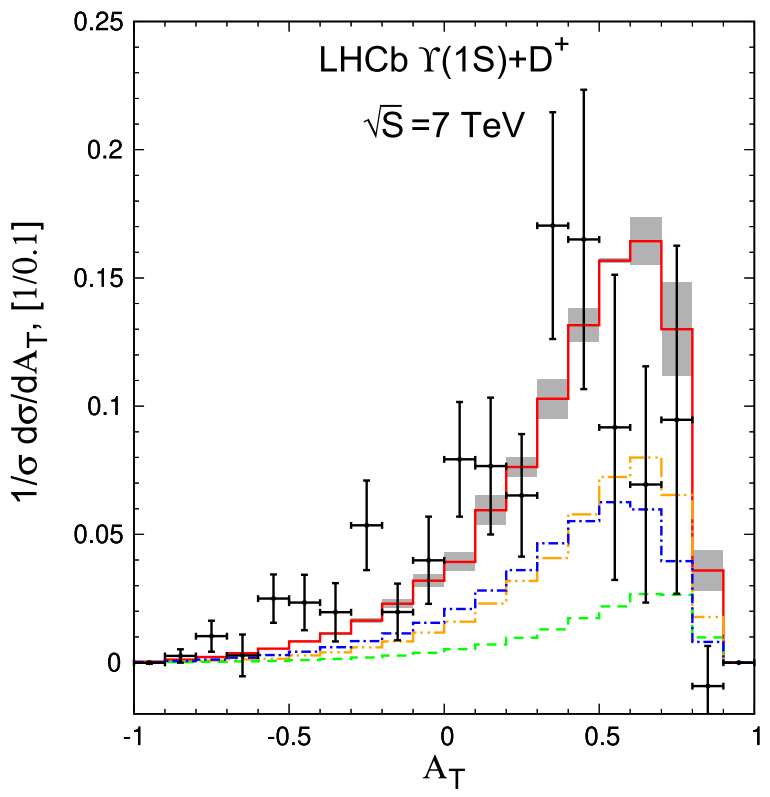

FIG. 7. (Left) Invariant mass spectrum of $\Upsilon(1 S) D^{+}$pairs. (Right) Transverse-momentum asymmetry $\mathcal{A}_{T}$ spectrum. Histograms are the same as in Fig. 2. LHCb data [5] are taken at $\sqrt{S}=7 \mathrm{TeV}, 2.0<y_{\Upsilon(D)}<4.5,0<p_{T \Upsilon}<15 \mathrm{GeV}$, and $1<p_{T D}<20 \mathrm{GeV}$. 
TABLE II. The total cross sections of $\Upsilon D^{+, 0}$ production at the $\mathrm{LHCb}$ for $\sqrt{S}=7 \mathrm{TeV}$.

\begin{tabular}{lcc}
\hline \hline & $\begin{array}{c}B_{\mu^{+} \mu^{-}} \times \sigma^{\Upsilon(1 S) D^{0}} \\
(\mathrm{pb})\end{array}$ & $\begin{array}{c}B_{\mu^{+} \mu^{-}} \times \sigma^{\Upsilon(1 S) D^{+}} \\
(\mathrm{pb})\end{array}$ \\
\hline $\begin{array}{l}\text { Direct contributions } \\
R+R \rightarrow \Upsilon\left[{ }^{3} S_{1}^{(1)}\right]+g\end{array}$ & 51 & 20 \\
$R+R \rightarrow \Upsilon\left[{ }^{3} S_{1}^{(8)}\right]+g$ & 37 & 15 \\
$\quad$ Sum of feed-down & 14 & 5 \\
$\quad$ contributions (5), (6) & 40 & 16 \\
Total cross section, & & $36_{-16}^{+19}$ \\
$\quad$ LO PRA & $91_{-41}^{+48}$ & $82 \pm 24$ \\
Total cross section, \\
$\quad$ experiment [5]
\end{tabular}

TABLE III. The cross sections of $\Upsilon D^{+, 0}$ production at the $\mathrm{LHCb}$ for $\sqrt{S}=8 \mathrm{TeV}$.

\begin{tabular}{lcc}
\hline \hline & $\begin{array}{c}B_{\mu^{+} \mu^{-}} \times \sigma^{\Upsilon(1 S) D^{0}} \\
(\mathrm{pb})\end{array}$ & $\begin{array}{c}B_{\mu^{+} \mu^{-}} \times \sigma^{\Upsilon(1 S) D^{+}} \\
(\mathrm{pb})\end{array}$ \\
\hline $\begin{array}{l}\text { Direct contributions } \\
R+R \rightarrow \Upsilon\left[{ }^{3} S_{1}^{(1)}\right]+g\end{array}$ & 61 & 24 \\
$R+R \rightarrow \Upsilon\left[{ }^{3} S_{1}^{(8)}\right]+g$ & 44 & 18 \\
$\quad$ Sum of feed-down & 17 & 6 \\
$\quad$ contributions (5), (6) & 49 & 19 \\
Total cross section, & & \\
$\quad$ LO PRA & $108_{-48}^{+56}$ & $42_{-19}^{+22}$ \\
Total cross section, \\
$\quad$ experiment [5]
\end{tabular}

$$
\begin{aligned}
& \left\langle\overline{\left.\left|\mathcal{M}\left[R R \rightarrow b \bar{b}+c\left(p_{c}\right)+\bar{c}\left(p_{\bar{c}}\right)\right]\right|^{2}\right\rangle}\right. \\
& \simeq \frac{2 g_{s}^{2}}{s_{c \bar{c}}} P_{q g}(z) \cdot \overline{\left.\mid \mathcal{M}\left[R R \rightarrow b \bar{b}+g\left(p_{c}+p_{\bar{c}}\right)\right)\right]\left.\right|^{2}},
\end{aligned}
$$

where $z=p_{c}^{0} /\left(p_{c}^{0}+p_{\bar{c}}^{0}\right), P_{q g}(z)=\left[z^{2}+(1-z)^{2}\right] / 2$, and angular brackets on the lhs stand for averaging over the azimuthal angle parametrizing the directions of momenta $\mathbf{p}_{c}$ and $\mathbf{p}_{\bar{c}}$ for constant $\mathbf{p}_{c}+\mathbf{p}_{\bar{c}}$ and $z$.

For the direct contributions from the subprocesses (12) we obtain $B_{\mu^{+} \mu^{-}} \times \sigma_{\text {direct }}^{D^{0}}\left[R+R \rightarrow b \bar{b}\left[{ }^{3} S_{1}^{(1+8)}\right]+c+\bar{c}\right]=$
$20 \mathrm{pb}$ at $\sqrt{S}=7 \mathrm{TeV}$ and $24 \mathrm{pb}$ at $\sqrt{S}=8 \mathrm{TeV}$; so for the ratio of direct cross sections we obtain

$$
\frac{\sigma_{\text {direct }}^{D^{0}}\left[R+R \rightarrow b \bar{b}\left[{ }^{3} S_{1}^{(1+8)}\right]+g\right]}{\sigma_{\text {direct }}^{D^{0}}\left[R+R \rightarrow b \bar{b}\left[{ }^{3} S_{1}^{(1+8)}\right]+c+\bar{c}\right]} \simeq 2.6 \div 2.5
$$

at $\sqrt{S}=7$ and $8 \mathrm{TeV}$. So our expectation of the dominant role of the gluon fragmentation in this process turns out to be correct.

\section{CONCLUSIONS}

In the present paper, we have demonstrated that the conclusion about DPS dominance in the total cross section for the process of associated hadroproduction of $\Upsilon$ and $D$ mesons, based on the LO collinear parton model calculations of Refs. [6,7] was premature, and one can relatively easily come up with the model that accounts more than half of the observed cross section. Also, due to the use of high-energy factorization with unintegrated PDFs dependent on transverse momenta of initial-state partons, the leading part of ISR corrections has been taken into account and all differential distributions are described reasonably well. Therefore, one has to be rather careful about the statement that DPS dominates the total cross section of relatively low-scale, hadronization-sensitive processes like the one considered in the present paper. Most likely, the solid evidence in favor of DPS can be obtained only from studies of differential distributions in specific regions of phase space and at high scales, where radiative corrections to the SPS contribution can be put under theoretical control.

\section{ACKNOWLEDGMENTS}

The authors thank the Ministry of Education and Science of the Russian Federation for financial support in the framework of the Samara University Competitiveness Improvement Program among the world's leading research and educational centers for 2013-2020, the task No. 3.5093.2017/8.9, and the Foundation for the Advancement of Theoretical Physics and Mathematics BASIS, Grant No. 18-1-1-30-1. We thank Professor B. A. Kniehl and Dr. Zhi-Guo He for stimulating discussions.
[1] M. Diehl, J.R. Gaunt, P. Plothl, and A. Schafer, Twoloop splitting in double parton distributions, arXiv: 1902.08019.

[2] M. Diehl and R. Nagar, Factorisation of soft gluons in multiparton scattering, J. High Energy Phys. 04 (2019) 124.
[3] M. Diehl, D. Ostermeier, and A. Schafer, Elements of a theory for multiparton interactions in QCD, J. High Energy Phys. 03 (2012) 089; Erratum 03 (2016) 1.

[4] E. Elias, K. Golec-Biernat, and A. M. Stasto, Numerical analysis of the unintegrated double gluon distribution, J. High Energy Phys. 01 (2018) 141. 
[5] R. Aaij (LHCb Collaboration), Production of associated $\Upsilon$ and open $\mathrm{p}$ charm hadrons in pp collisions at $\sqrt{S}=7$ and $8 \mathrm{TeV}$ via double parton scattering, J. High Energy Phys. 07 (2016) 052.

[6] A. V. Berezhnoy and A. K. Likhoded, Associated production of $\Upsilon$ and open charm at LHC, Int. J. Mod. Phys. A 30, 1550125 (2015).

[7] A. K. Likhoded, A. V. Luchinsky, and S. V. Poslavsky, Production of associated $\chi_{b}$ and open charm ar the LHC, Phys. Lett. B 755, 24 (2016).

[8] M. A. Nefedov, V. A. Saleev, and A. V. Shipilova, Dijet azimuthal decorrelations at the LHC in the parton Reggeization approach, Phys. Rev. D 87, 094030 (2013).

[9] M. A. Nefedov, A. V. Karpishkov, and V. A. Saleev, $B \bar{B}$ angular correlations at the LHC in the parton Reggeization approach merged with higher-order matrix elements, Phys. Rev. D 96, 096019 (2017).

[10] G. T. Bodwin, E. Braaten, and G. P. Lepage, Rigorous QCD analysis of inclusive annihilation and production of heavy quarkonium, Phys. Rev. D 51, 1125 (1995).

[11] M. A. Nefedov, V. A. Saleev, and A. V. Shipilova, Prompt $\Upsilon(n S)$ production at the LHC in the Regge limit of QCD, Phys. Rev. D 88, 014003 (2013).

[12] B. A. Kniehl, G. Kramer, I. Schienbein, and H. Spiesberger, Reconciling Open-Charm Production at the Fermilab Tevatron with QCD, Phys. Rev. Lett. 96, 012001 (2006).

[13] A. Karpishkov, V. Saleev, and A. Shipilova, Large-pT production of $\mathrm{D}$ mesons at the $\mathrm{LHCb}$ in the parton Reggeization approach, Phys. Rev. D 94, 114012 (2016).

[14] R. Maciula, V. A. Saleev, A. V. Shipilova, and A. Szczurek, New mechanisms for double charmed meson production at the LHCb, Phys. Lett. B 758, 458 (2016).

[15] M. A. Nefedov and V. A. Saleev, On the one-loop calculations with Reggeized quarks, Mod. Phys. Lett. A 32, 1750207 (2017); M. A. Nefedov, Computing one-loop corrections to effective vertices with two scales in the EFT for Multi-Regge processes in QCD, arXiv:1902.11030.

[16] M. A. Nefedov and V. A. Saleev, From LO to NLO in the parton Reggeization approach, EPJ Web Conf. 191, 04007 (2018).

[17] L. N. Lipatov, Gauge invariant effective action for high energy processes in QCD, Nucl. Phys. B452, 369 (1995).
[18] L. N. Lipatov and M. I. Vyazovsky, Quasi-multi-Regge processes with a quark exchange in the t-channel, Nucl. Phys. B597, 399 (2001).

[19] E. N. Antonov, L. N. Lipatov, E. A. Kuraev, and I. O. Cherednikov, Feynman rules for effective Regge action, Nucl. Phys. B721, 111 (2005).

[20] M. A. Kimber, A. D. Martin, and M. G. Ryskin, Unintegrated parton distributions, Phys. Rev. D 63, 114027 (2001).

[21] V. A. Saleev and A. V. Shipilova, Inclusive b-jet and bb-dijet production at the LHC via Reggeized gluons, Phys. Rev. D 86, 034032 (2012).

[22] A. van Hameren, P. Kotko, and K. Kutak, Helicity amplitudes for high-energy scattering, J. High Energy Phys. 01 (2013) 078.

[23] A. van Hameren, K. Kutak, and T. Salwa, Scattering amplitudes with off-shell quarks, Phys. Lett. B 727, 226 (2013).

[24] A. van Hameren, KaTie: For parton-level event generation with kT-dependent initial states, Comput. Phys. Commun. 224, 371 (2018).

[25] B. Mele and P. Nason, The fragmentation function for heavy quarks in QCD, Nucl. Phys. B361, 626 (1991).

[26] M. Juszczak, R. Maciula, and A. Szczurek, Production of two $c \bar{c}$ pairs in double-parton scattering, Phys. Rev. D 85, 094034 (2012).

[27] B. A. Kniehl, D. V. Vasin, and V. A. Saleev, Charmonium production at high energy in the $\mathrm{k}(\mathrm{T})$-factorization approach, Phys. Rev. D 73, 074022 (2006).

[28] P. L. Cho and A. K. Leibovich, Color octet quarkonia production. 2, Phys. Rev. D 53, 6203 (1996); Color octet quarkonia production, Phys. Rev. D 53, 150 (1996).

[29] T. Hahn, Generating Feynman diagrams and amplitudes with FEynArts 3, Comput. Phys. Commun. 140, 418 (2001).

[30] R. Mertig, M. Bohm, and A. Denner, FEYN CALC: Computer algebraic calculation of Feynman amplitudes, Comput. Phys. Commun. 64, 345 (1991).

[31] C. Patrignani et al. (Particle Data Group), Review of particle physics, Chin. Phys. C 40, 100001 (2016).

[32] R. Aaij et al. (LHCb Collaboration), Measurements of prompt charm production cross-sections in pp collisions at $\sqrt{S}=13 \mathrm{TeV}$, J. High Energy Phys. 03 (2016) 159.

[33] A. D. Martin, W. J. Stirling, R. S. Thorne, and G. Watt, Parton distributions for the LHC, Eur. Phys. J. C 63, 189 (2009). 